\title{
Multimodality Deformable Registration of Pre- and Intraoperative Images for MRI-Guided Brain Surgery
}

\author{
Nobuhiko Hata ${ }^{1,2}$, Takeyoshi Dohi ${ }^{2}$, Simon Warfield ${ }^{1}$, William Wells III ${ }^{1,3}$ \\ Ron Kikinis', Ferenc A. Jolesz' \\ ${ }^{1}$ Image-guided Therapy Program, Department of Radiology \\ Brigham and Women's Hospital and Harvard Medical School \\ 75 Francis St., Boston, MA 02115, USA \\ \{noby, simonw, sw, kikinis, jolesz\}@bwh.harvard.edu \\ ${ }^{2}$ Department of Precision Machinery Engineering, Faculty of Engineering \\ dohi@miki.pe.u-tokyo.ac.jp \\ ${ }^{3}$ Artificial Intelligence Laboratory, Massachusetts Institute of Technology
}

\begin{abstract}
A method by which to register multimodality medical images accommodating soft tissue deformation is presented in the context of interventional therapy with a MR scanner. Accuracy testing with arbitrarily deformed MR images and application studies of a pig's brain were undertaken to evaluate the feasibility of the method. When Mutual Information is employed as the voxel similarity measure in the matching energy function, the algorithm can accommodate multimodality images. Coupled with rigid registration, the deformable registration of pre- and intraoperative multi-modality images enables surgeons to precisely define critical anatomical structures, such as vessels and functional areas, and to localize and optimize trajectories. The method directly and automatically works on volumetric multimodality images. Thus the algorithm is suitable for intraoperative registration, where stability and simplicity are desirable.
\end{abstract}

\section{Motivation}

The advantages of intraoperative MRI [1] as a guidance tool have been well acknowledged in neurosurgical procedures [2], including biopsy [3], drainage, and tumor resection in which the aim is to perform the least invasive surgery possible with MRI-specific imaging capabilities (multiplane imaging, soft tissue discrimination and temperature mapping). Despite its obvious advantages in terms of accessibility, the surgical requirements for interventional MRI limit its imaging capability in comparison to that of conventional diagnostic MRI.

Our proposal to overcome this problem as detailed elsewhere [4] is the rigid registration of preoperative images to intraoperative MR images. Images obtained by preoperative computed tomography (CT), T1- and T2-weighted MRI, magnetic resonance angiography (MRA), and single photon emission computed tomography (SPECT) are registered to the intraoperative MR images, in order to show the preoperative anatomical and pathological tissue discrimination in the interventional field. This approach also permits simultaneous access to various preoperative 
scans and intraoperative images. The incorporation is achieved by the rigid multimodality registration algorithm "Maximization of Mutual Information" $[5,6]$.

Provided that the patient's position with respect to the scanner is stable, the geometric relation between the (preoperatively defined) master space and the intraoperative scanner coordinate system remains fixed. This assumption is valid early in part brain surgery, since patients are fixed to the MRI table with a Mayfield clamp.

However, as Kato et al. have reported [7], the brain deforms during surgery, primarily because of cerebrospinal fluid leakage and tissue resection. In our experience intraoperative MRI has revealed that the brain shifts at most approximately $17 \mathrm{~mm}$ after craniotomy, biopsy, and tumor resection.

This problem motivated us to work on a deformable registration to accommodate any deformations that occur in soft tissues. The deformable registration method developed should be fully automated and accurate in multimodality image settings.

\section{Deformable registration with "Maximization of Mutual Information"}

\subsection{Definition of the problem}

Deformable registration using a correlation-based approach divides a volume into subvolumes, searching for optimal translation for each subvolume $p=(x, y, z)^{T} \in \mathfrak{R}^{3}$. Several groups of investigators [8-12] have reported that the energy function measuring goodness-of-matching can be formulated as the sum of a voxel similarity and an elastic regularization energy, i.e.

$$
F(d)=\sum_{n=1}^{N} \int_{\Omega}-I(r(p), s(p+d)) d x+\omega U(d),
$$

where $d$ denotes displacement of a subvolume $p$, or with a transformation function: $T(p)=p(x, y, z)^{T}+d(u, v, w)^{T}$.

In equation (1), I() counts a similarity measure of a subvolume $p$ in the reference data $r$ and the sample data $s$. The second term $U()$ denotes a regularization energy, which imposes a local elasticity constraint on displacement of subvolumes. $\omega$ is a weighing coefficient that controls the level of the regularization, or elasticity, effect.

The general problem of deformable registration is to find a set of $d$ that minimizes the matching energy function $F()$.

\subsection{Optimization of matching energy function}

We employ a stochastic gradient search approach for the optimization of matching energy (1). The local maximum of $F$ is computed with a gradient descent optimization scheme incorporating many steps: 


$$
T_{n} \leftarrow T_{n-1}+\lambda \frac{d F}{d T}=T_{n-1}+\lambda \frac{d}{d T}(-I+\omega U)=-\frac{d I}{d T}+\omega \frac{d U}{d T}
$$

where $\lambda$ is a step size parameter. At each step new samples $r$ and $s$ are drawn based on $T_{n}$, and the steps are taken until convergence is obtained.

\subsection{Mutual information as a voxel similarity measure}

We employed Mutual information [13] as the voxel similarity measure in the right term of (1). Mutual information was introduced by Viola and Wells for multimodality medical image registration $[5,6]$. This method works automatically and directly on medical images, in contrast to other methods that require the setting of fiducial markers or some other types of manual interaction for registration. Thus, the algorithm is suitable for intraoperative registration, where stability and simplicity are desirable.

Mutual information is formulated as

$$
I(r(x), s(T(x))) \equiv H(r(x))+H(s(T(x)))-H(r(x), s(T(x))),
$$

where $r(x)$ and $s(x)$ denote observations of the reference data and the sample data, respectively, and $x$ represents the coordinates of the voxel treated as a random variable over coordinate locations in the reference data. $H(\cdot)$, the entropy of a random variable, is formulated as

$$
H(A)=-\int_{-\infty}^{\infty} P(z) \ln P(z) d z=-\frac{1}{N_{A}} \sum_{z_{i} \in \Omega_{A}} \log P\left(z_{i}\right),
$$

which represents an expectation of the negative logarithm of the probability density $P(z)$ of the observation $A$. The probability $P(z)$ is computed from a histogram of an image $r$ or $s$, or from a joint histogram of $r$ and $s$ in which each voxel contains two pixel values from $r$ and $s$. Thus the dimension of the joint histogram is two, while that of a single histogram is one.

We will construct the derivative of the Mutual Information in (2) with respect to the transformation variable $T$ in order to use it in stochastic optimization:

$$
\frac{d I}{d T}=\sum_{i \in \Omega_{A}}\left(\frac{\partial I}{\partial s_{i}} \frac{d s_{i}}{d T}+\frac{\partial I}{\partial r_{i}} \frac{d r_{i}}{d T}\right)=\sum_{i \in \Omega_{A}}\left(\frac{\partial I}{\partial s_{i}} \frac{d s_{i}}{d T}\right)
$$

where the term $\frac{d s_{i}}{d T}$ is the gradient of the image intensity of a voxel in the sample data, with respect to transformation $T$. In the simple case where $T$ is a linear operator [14], (5) is then formulated as follows:

$$
\frac{d I}{d T}=-\frac{1}{N} \sum_{i \in \Omega_{A}}\left(\frac{1}{P\left(s_{i}\right)} \frac{\partial P\left(s_{i}\right)}{\partial s}-\frac{1}{P\left(r_{i}, s_{i}\right)} \frac{\partial P\left(r_{i}, s_{i}\right)}{\partial s}\right) \nabla s\left(T\left(x_{i}\right)\right) x_{i}^{T} .
$$




\subsection{Elastic energy as regularization energy measure}

With the application of external force, an object deforms until equilibrium is reached at which point external force and internal stress are balanced. At the same, time the stress causes strain or displacement, which eventually causes the object to deform. If we hypothesize that the object deforms only elastically (and not plastically), we can assume a potential energy that measures the work by the external force. In an ideal situation in which the object deforms elastically, this potential energy should be minimized according to the principle of minimum potential energy [15].

Elastic deformation principles [15] introduce other terms: Young's modulus $E$, shear modulus of elasticity $G$, shear strain $\gamma$. If we assume $\gamma=0$, the derivative of elastic energy $U$ with respect to transformation $T$ of a subvolume $p(x, y, z)$ is formulated as

$$
\begin{aligned}
\frac{d U}{d T} & =\nabla_{T} U \\
& =\left(\frac{E}{2}+2 G\right)\left(2 \frac{\partial u}{\partial x} \frac{\partial^{2} u}{\partial x^{2}}+2 \frac{\partial v}{\partial y} \frac{\partial^{2} v}{\partial y^{2}}+2 \frac{\partial w}{\partial z} \frac{\partial^{2} w}{\partial z^{2}}\right) \\
& +(E+G)\left(\frac{\partial^{2} u}{\partial x^{2}}\left(\frac{\partial v}{\partial y}+\frac{\partial w}{\partial z}\right)+\frac{\partial^{2} v}{\partial y^{2}}\left(\frac{\partial u}{\partial x}+\frac{\partial w}{\partial z}\right)+\frac{\partial^{2} w}{\partial z^{2}}\left(\frac{\partial u}{\partial x}+\frac{\partial v}{\partial y}\right)\right)
\end{aligned}
$$

\section{Results}

\subsection{Experimental overview}

In the first experiment, the evaluated the accuracy of the algorithm by synthesizing sample images from the reference (target) images. Matching error was estimated on the basis of the hypothesis that perfect registration would deform the synthesized image back to its original shape (reference image).

In the second experiment, we evaluated the feasibility of the algorithm, registering MRI scans of a pig's brain before and after a clay weight was placed on the brain.

\subsection{Accuracy test: preoperative MRI vs. synthesized intraoperative MRI}

The first study registered synthesized deformed images with the original image and numerically compared the result with the known data. The original data were obtained by T1-weighted MRI (3D SPGR, TR/TE $=35 / 5$, Sagital, $256^{2}$ pixels $\times 124$ slices, $0.78125^{2} \times 1.5 \mathrm{~mm}$ ) with contrast agent (Gadolinium-DTP Areg).

Voxels were displaced in a normal distribution. Given the center of force in the image at $\left(x_{0}, y_{0}, z_{0}\right)$, a displacement $(u, v, w)$ at $\left(x_{0}+d x, y_{0}+d y, z_{0}+d z\right)$ was

$$
(u, v, w)=\left(k \frac{1}{\sqrt{2 \pi}} e^{-d x^{2} / 2}, k \frac{1}{\sqrt{2 \pi}} e^{-d y^{2} / 2}, k \frac{1}{\sqrt{2 \pi}} e^{-d z^{2} / 2}\right)
$$


where $k$ is a constant coefficient to magnify the displacement.

Comparing the result with known data, root-mean-square and standard deviation of the error along the sagital (RL-), colonal (AP-) and axial (SI-) axis's are summarized in Table 1.

The initial displacement of the pixels, $3.39 \pm 2.72 \mathrm{~mm}$ (in-plane direction), corrected by the deformable registration with an error of $1.58 \pm 0.91 \mathrm{~mm}$, or double the voxel size of $0.78125^{2} \mathrm{~mm}$. A similar result was obtained in the outplane direction.

A coarse-to-fine multiresolution scheme was employed in direct descending optimization to avoid a matching energy value in the local minimal. The resolution was changed from $1 / 8,1 / 4,1 / 2$ to the original size $\left(256^{2}\right.$ pixel $x 124$ slices). Ten iterations were performed in each resolution. The parameters given were $\omega=2.0$ and $\lambda=100000$, and the computation time was approximately 21 minutes by UltraSPARC 2 (dual 200MHz, 256MB, SUN Microsystems, Mountain View, CA).

Table 1: Result of accuracy testing with synthesized deformed T1-weighted MRI.

\begin{tabular}{lcc}
\hline & Displacement [mm] & Registration error [mm] \\
\hline $\begin{array}{l}\text { In-plane direction } \\
\text { (AP, IS-axis) }\end{array}$ & $3.39 \pm 2.72$ & $1.58 \pm 0.91$ \\
$\begin{array}{l}\text { Out-plane direction } \\
\text { (LR-axis) }\end{array}$ & $4.75 \pm 3.13$ & $2.44 \pm 1.21$ \\
\hline
\end{tabular}

\subsection{Animal study: deformed vs. original pig brain MRI}

In the second experiment, we undertook the deformable registration of MRI scans of a pig's brain.

After anesthesia (isoflurane $2 \%$ and oxygen $5 \mathrm{~L}$ ) and $\mathrm{KCL} 20 \mathrm{ml}$ injection, the pig was decapitated. Craniotomy was performed to open a bur hole (approximately 100 $\mathrm{mm}$ in diameter) at the top of the head and the brain surface was exposed after the dura had been partially removed. The head was rigidly fixed in a coil for brain scan and placed in the gantry of a MR scanner (0.5T, MRH-500, Hitachi Medico, Japan). After the fixation of the brain in a coil, the initial T1-GFE scan (TR/TE 60/16, FOV $128 \mathrm{~mm}, 128 \times 128 \times 64$ pixels, $1.0 \times 1.0 \times 1.5 \mathrm{~mm} /$ voxel, data acquisition 4 times) was performed.

Next, the pressure was applied to upper left section of the brain with a specially developed acrylic bar with clay at the top. MRI scans were performed with a weight of $20.3 \mathrm{~g}$ and then $30.1 \mathrm{~g}$ (Figure 1).

The deformable registration was applied to fuse (1) the initial scan without the weight vs. The second scan made after placing of the first weight (total, $20.3 \mathrm{~g}$ ), and (2) the initial scan vs. The third scan after placing the additional weight (total, 30.1 g). The latter result is presented in Figure 2. 


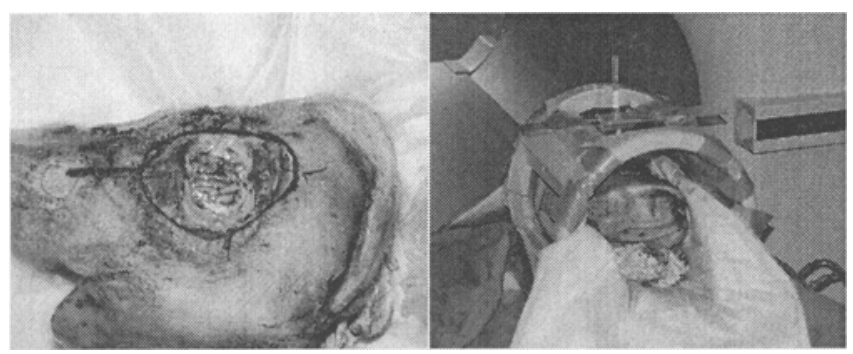

Figure 1: [Left] Bur hole approximately $100 \mathrm{~mm}$ in diameter was made to expose the brain of a pig (male, $48.5 \mathrm{~kg}, 100$ days old). [Right] A specially developed acrylic frame with a clay weight was fixed to the MRI head coil, which also rigidly holds the pig's head.
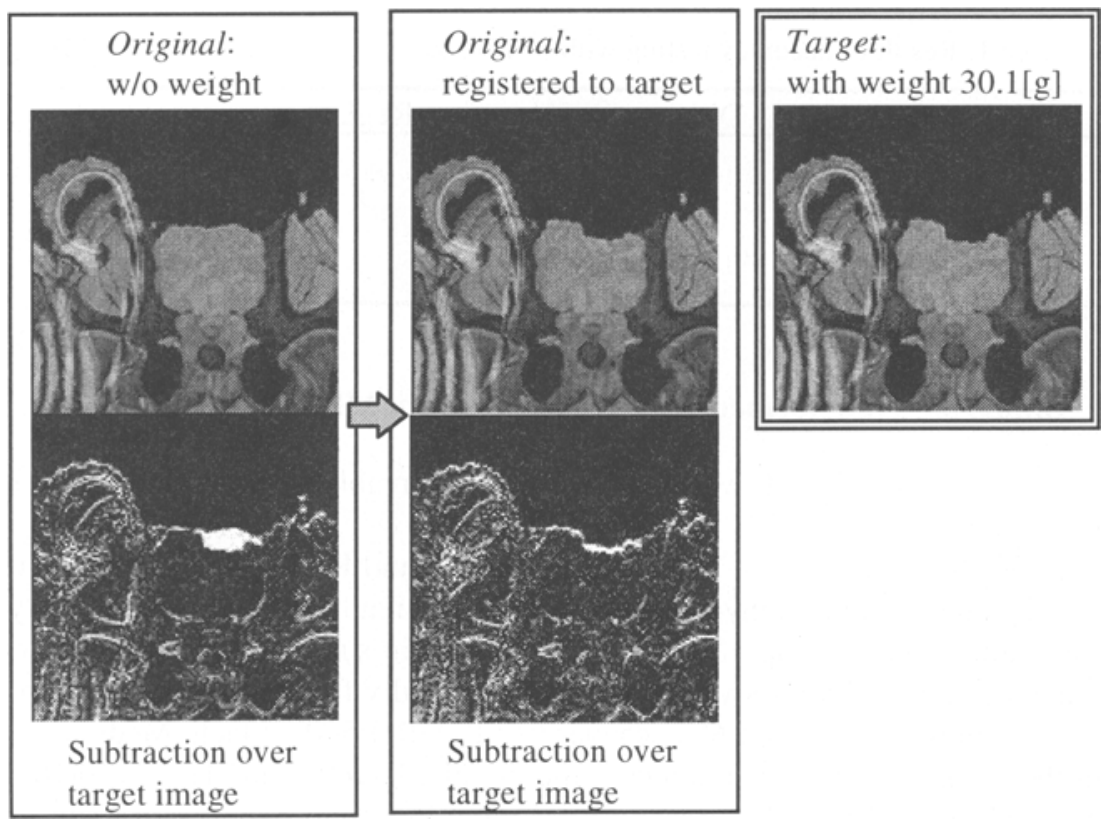

Figure 2: Registered MR images of a pig's brain. The target images are scanned while pushing the top of the brain with a weight $(20.3 \mathrm{~g})$. The original MRI is registered to the target image allowing elastic deformation.

As in the first experiment, a coarse-to-fine multiresolution scheme was employed in optimization to avoid a matching energy value in local minimal; the resolution was changed from 1/8, 1/4, 1/2 the original size of $128^{2}$ pixels $\times 64$ slices; and 10 iterations were performed in each resolution. The parameters given were $\omega=2.0$ 
and $\lambda=100000$ and the computation time was approximately 11 minutes by UltraSPARC 2 (dual 200MHz, 256MB, SUN Microsystems, Mountain View, CA).

\section{Discussion}

In application of MRI-guided therapy, deformable registration must deal with a variety of imaging modalities and protocols. For instance, the direct deformable registration of SPECT and MRI will superimpose the tumor tissue characterization onto the intraoperative images. Therefore, the voxel similarity measure $I()$ in (1) should be carefully selected, as in rigid registration.

Most previously reported methods of deformable registration have used a regularized pixel value, or root mean square, as the voxel similarity measure $[8,10$, 11, 16]. This approach works fine if we can restrict the image modalities (e.g., anatomical template, MRI, and CT) and can approximate the intensity value of tissues.

In the next stage of our investigations, we will examine the application of the method to a multimodality setting, accuracy testing with a single-modality image (T1-weighted MRI).

As reported elsewhere [4], we have set up a surgical navigation system for MRIguided therapy that rigidly registers preoperative images to intraoperative images by the "Maximization of Mutual Information" method [5, 6]. Guided by multiple modalities (T1- and T2-weighted MRI and intraoperative MRI), surgeons and interventional radiologists can localize and optimize trajectories. The approaching path is evaluated by interactively choosing the scan plane with an optical locator device and re-slicing the registered multimodality preoperative images, with a refresh rate of $29 \mathrm{~Hz}$. The registration of preoperative T1-weighted MRI to intraoperative T1-weighted images takes approximately 9 minutes.

In the future, the deformable registration technique presented in this paper will be implemented in this system to accommodate the soft tissue deformation, which cannot be tracked by rigid registration alone.

\section{Acknowledgment}

Nobuhiko Hata was supported in part by Japan Society for the Promotion of Science, William Wells III by the The Whitaker Foundation, Ron Kikinis and Ferenc A. Jolesz by NIH grant P01-CA67165.

\section{References}

[1] Schenck, J. F., Jolesz, F. A., Roemer, P. B., Cline, H. E., Lorensen, W. E., Kikinis, R., Silverman, S. G., Hardy, C. J., Barber, W. D., Laskaris, E. T., and et al.: Superconducting open-configuration MR imaging system for image-guided therapy. Radiology 195 (1995) $805-814$

[2] Black, P. M., Moriarty, T., Alexander, E., 3rd, Stieg, P., Woodard, E. J., Gleason, P. L., Martin, C. H., Kikinis, R., Schwartz, R. B., and Jolesz, F. A.: Development and 
implementation of intraoperative magnetic resonance imaging and its neurosurgical applications. Neurosurgery 41 (1997) 831-845

[3] Moriarty, T. M., Kikinis, R., Jolesz, F. A., Black, P. M., and Alexander III, E.: Magnetic resonance imaging therapy. Intraoperative MR imaging. Neurosurg Clin N Am 7 (1996) 323-331

[4] Hata, N., Dohi, T., Kikinis, R., Jolesz, F., and Wells III, W. M.: Computer assisted intra-operative MR-guided therapy: pre- and intra-operative image registration, enhanced three-dimensional display, deformable registration. 7th Annual meeting of Japan Society of Computer Aided Surgery, Sapporo, Japan, (1997) 119-120

[5] Viola, P. A.: Alignhment by Maximization of Mutual Information. Artificial Intelligence Laboratory. Cambridge, MA: Massachusetts Institute of Technology (1995) 155.

[6] Wells III, W. M., Viola, P., Atsumi, H., Nakajima, S., and Kikinis, R.: MultiModal Volume Registration by Maximization of Mutual Information. Medical Image Analysis 1 (1996) 35-51

[7] Kato, A., Yoshimine, T., Hayakawa, T., Tomita, Y., Ikeda, T., Mitomo, M., Harada, K., and Mogami, H.: A frameless, armless navigation system for computer-assisted neurosurgery. J Neurosurg 74 (1991) 845-849

[8] Bajcsy, R. and Kovacic, S.: Multiresolution elastic matching. Comp Vis Graph Image Proc 46 (1989) 1-21

[9] Gee, J. C., Reivich, M., and Bajcsy, R.: Elastically deforming 3D atlas to match anatomical brain images. J of Compt Assist Tomogr 17 (1993) 225-236

[10] Collins, D. L., Peters, T. M., Dai, W., and Evans, A. C.: Model based segmentation of individual brain structures from MRI data. Visualization in Biomedical Computing, Chapell Hill, NC, (1992) 10-23

[11] Christensen, G. E., Rabbitt, R. D., and Miller, M. I.: 3D brain mapping using a deformable neuroanatomy. Physics in Medicine and Biology 39 (1994) 609-618

[12] Miller, M. I., Christensen, G. E., Amit, Y., and Grenander, U.: Mathematical textbook of deformable neuroanatomies. National Academy of Sciences, (1993) 1194411948

[13] Papoulis, A.: Probability, Random Variables, and Stochastic Processes, Third Edition. McGraw-Hill,New York, NY (1991)

[14] Wells III, W. M., Halle, M., Kikinis, R., and Viola, P.: Alignment and tracking using graphics hardware: demonstration with software. RSNA annual meeting, Chicago, IL, (1996)

[15] Washizu, K.: Variational methods in elasticity and plasticity. Pergamon Press, Headington Hill Hall, Oxford (1968)

[16] Christensen, G. E., Miller, M. I., and Vannier, M.: A 3D deformable magnetic resonance textbook based on elasticity. Applications of computer vision in medical image processing, Stanford Univ., (1994) 153-156

[17] West, J., Fitzpatrick, J. M., Wang, M. Y., Dawant, B. M., Maurer, C. R. J., Kessler, R. M., Maciunas, R. J., Barillot, C., Lemoine, D., Collignon, A., Maes, F., Suetens, P., Vandermeulen, D., van den Elsen, P. A., Napel, S., Sumanaweera, T. S., Harkness, B., Hemler, P. F., Hill, D. L., Hawkes, D. J., Studholme, C., Maintz, J. B., Viergever, M. A., Malandain, G., Woods, R. P., and al., e.: Comparison and evaluation of retrospective intermodality brain image regsistraion techniques. J Comput Assist Tomogr 21 (1997) 554566 